\title{
Bacteriology and Antibiotic Susceptibility Pattern of Peritonsillar Abscess
}

\author{
Acharya A, 'Gurung R, ${ }^{1}$ Khanal B, ${ }^{1}$ Ghimire A \\ 'Department of Microbiology, ${ }^{2}$ Department of Otolaryngology and Head-Neck Surgery, \\ BPKIHS, Dharan, Nepal.
}

\section{ABSTRACT}

Introduction: Peritonsillar abscess is a common complication of acute tonsillitis. Its management consists of aspiration or surgical drainage followed by appropriate antibiotics. Appropriate antibiotic treatment depends on the common organisms associated with infection and their antibiotic sensitivity pattern in local scenario.

Methods: Pus samples aspirated from diagnosed cases of peritonsillar abscess and submitted for culture and sensitivity in last two years were included in this study. Identification of different microorganisms was made on the basis of the microscopic findings, observation of their colony morphology and standard biochemical reactions. Susceptibility pattern to commonly used antibiotics were determined by Clinical Laboratory Standards Institute (CLSI) guidelines.

Results: Altogether 24 pus samples were included in the study. Positive culture was obtained from 18 samples. Among them one organism was isolated from 13 samples whereas from five samples multiple organisms were isolated. From six samples no organisms could be cultured. Altogether Streptococcus pyogenes was isolated from 12 samples and Staphylococcus aureus from five samples. Other isolated organisms included Haemophlilus influenzae, Pseudomonas aeruginosa, Escherichia coli and Enterococcus species. Penicillin was effective for Streptococcus pyogenes whereas Staphylococcus aureus was resistant to it. Cloxacillin was found to be effective for Staphylococcus aureus. Ciprofloxacin and Ceftazidime were found to be effective for both organisms.

Conclusions: Streptococcus pyogenes and Staphylococcus aureus were more commonly associated with peritonsillar abscess. Streptococcus pyogenes were sensitive to penicillin but all Staphylococcus aureus were resistant to it. This fact should be considered in clinical practice for management of peritonsillar abscess.

Key Words: Bacteriology, Peritonsillar abscess, Susceptibility pattern

Correspondence:

Anju Acharya

Department of Microbiology

BPKIHS, Dharan, Nepal.

Email: anju_71np@yahoo.com

Phone: 9804328812 


\section{INTRODUCTION}

Peritonsillar abscess (PTA) refers to a collection of pus between the fibrous capsule of the pharyngeal tonsil and the superior constrictor muscles of the pharynx. It is the most common site of head and neck infection in adults. ${ }^{1,2}$ The incidence of PTA in the United States is about 30 per 100,000 person-years. ${ }^{3}$ In United Kingdom, it averages 13 adult cases per 100,000 person-years. ${ }^{4}$ No such data is available for Nepal.

The proper treatment for PTA inczazaludes aspiration or drainage of pus followed by administration of appropriate antibiotics. Appropriate antimicrobial therapy may reduce the potential for complications. ${ }^{5}$ Choice of appropriate antibiotics depends on common organisms and their antimicrobial susceptibility pattern.

The purpose of the present study was to find out the common organism associated with peritonsillar abscess and their antimicrobial susceptibility pattern in Eastern Nepal.

\section{METHODS}

A prospective study was designed which evaluated samples of pus aspirated from diagnosed cases of peritonsillar abscess and submitted for bacteriological culture and sensitivity to microbiology unit of clinical laboratory services (CLS) of B.P Koirala Institute of Health Sciences from January 1, 2007 to December 31, 2008.

After Gram staining of all the samples, direct microscopic examination was performed. All specimens were cultured on blood agar, MacConkey agar, and chocolate agar then incubated at $37^{\circ} \mathrm{C}$ for 24 to 48 hours. Presumptive identification of different microorganisms was made based on the microscopic findings and observation of their colony morphology. Final identification was made by standard biochemical tests. ${ }^{6,7}$

Antimicrobial susceptibility test to commonly used antimicrobial agents was performed on Muller Hinton agar by the Kirby Bauer disc diffusion method recommended by the Clinical Laboratory Standards Institute (CLSI) guidelines. The results were interpreted according to the CLSI criteria. ${ }^{8} \mathrm{CLSI}$ criteria depends on the sizes of the 'Zones of Inhibition' to categorize the levels of susceptibility of organisms to various antimicrobial agents. These criteria are developed by comparing the zone diameters to Minimum Inhibitory Concentrations (MICs) of a large number of isolates, relevant to the particular class of drug.

\section{RESULTS}

The study population consisted of seven males and 17 female patients with peritonsillar abscesses. The clinical records of the patients were analysed. Their ages ranged from seven years to 75 years with a mean age of 30.3 years. Third decade was the most commonly affected age (Figure 1).

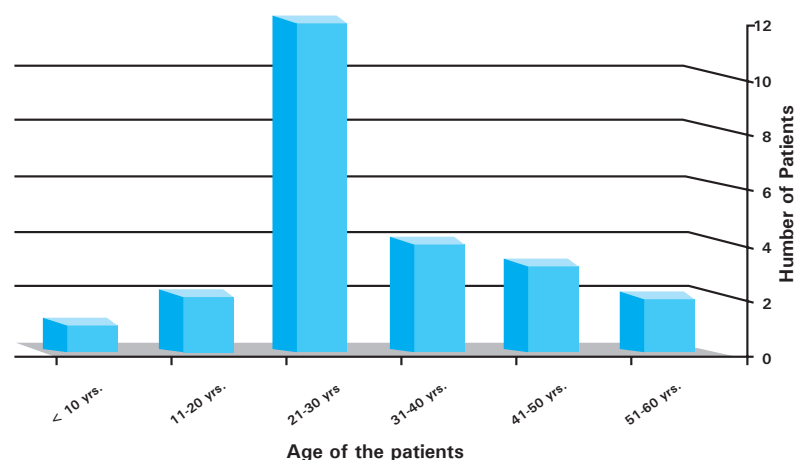

Figure 1. Distribution of age of the patients

Positive cultures were obtained from 18 (75\%) samples. Only one organism was isolated from 13 samples whereas from five samples more than one organism was isolated. Among the positive cultures, Streptococcus pyogenes was isolated from 12 samples followed by Staphylococcus aureus which was isolated from five samples. Other isolated organisms included Haemophlilus influenzae, Pseudomonas aeruginosa, Escherichia coli and Enterococcus species. (Figure 2). No organism could be cultured from six samples.

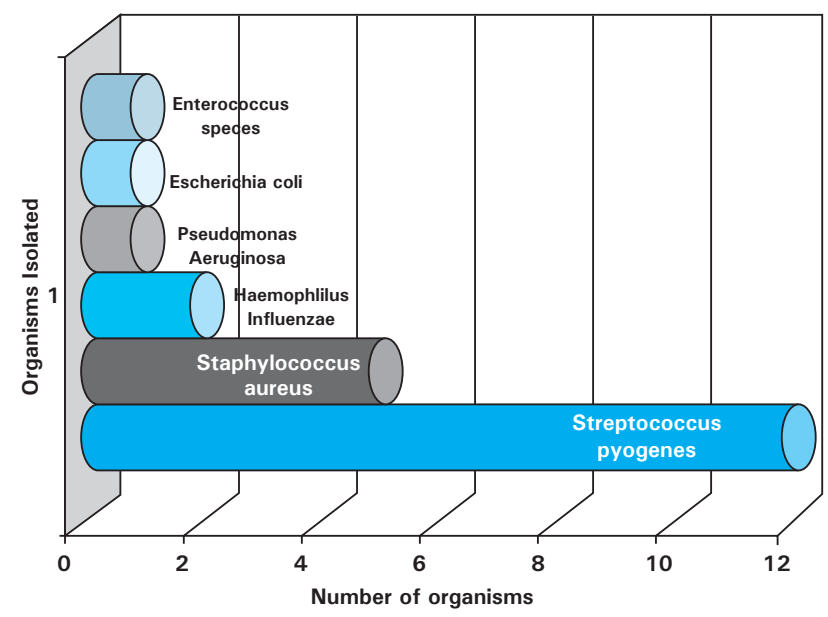

Figure 2. Organisms Isolated

Ciprofloxacin and Ceftazidime were found to be effective for both Streptococcus pyogenes and Staphylococcus aureus. Cephotaxim was more effective for Streptococcus pyogenes than Staphylococcus aureus, whereas Cloxacillin was only effective for Staphylococcus aureus and Penicillin was only effective for Streptococcus pyogenes (Figure 3). 


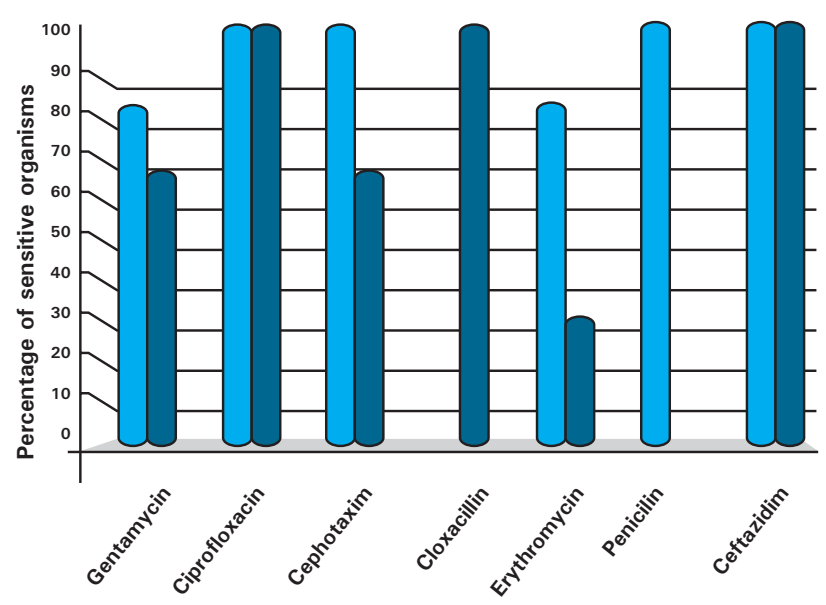

Antibiotics

Streptococcus pyogenes Straphylococcus aureus

Figure 3. Sensitivity patterns of the organisms

\section{DISCUSSION}

Peritonsillar abscess (PTA) is a disease usually affecting young adults. The majority of patients are aged between 10 and 40 years. ${ }^{9,10}$ This occurs rarely in infants and children. Young adults (21-30 Years of age) were the most commonly affected age group in this study as well. In our study females preponderance was observed.

In our study $75 \%$ samples yielded positive culture, which is less than that of Maharaj et al and Jokipii et al who had positive rates of $85 \%$ and $88.1 \%$ respectively. ${ }^{5,11}$ However it is more than the results obtained by Hall with less than $50 \%$ and Snow et at with only sixty percent. ${ }^{12,13}$ Relatively low culture positive results in the present study is mainly due to the fact that the facility of anaerobic culture is not available in our centre. Consumption of antibiotics prior to the aspiration of PTA could be another factor responsible for negative cultures.

There is considerable variation in the literature with regard to the bacteriology of peritonsillar abscess. The results of the studies depend on geographical and time variations, various local factors and the techniques used for the study. That is why common microorganisms for a particular disease and its sensitivity pattern to commonly used antibiotics in a local scenario are very important for the effective management of the disease.

Among the total positive culture samples the most commonly isolated organism in our study was Streptococcus pyogenes, which was 67\% (12 of 18) followed by Staphylococcus aureus (28\%). The results obtained by Brook and Jokipii et al are similar to our results. ${ }^{11,14}$

The treatment of peritonsillar abscess requires both the selection of appropriate antibiotics and the best procedure to remove the abscessed material. Individualized treatment modalities based on the microbiological study of the aspirate will result in more successful outcomes.

In present study all Streptococcus pyogenes were sensitive to Penicillin but all Staphylococci were resistant to penicillin which was isolated in $28 \%$ of cases. That means, about $1 / 3$ of the isolated organisms were resistant to penicillin. It may be due to the production of betalactamase enzyme. Beta-lactamase-producing organisms were isolated in $18.9 \%$ by Mitchelmore et al and in $52 \%$ by Brook et al from peritonsillar abscess. ${ }^{15,16}$

In present study both organisms were $100 \%$ sensitive to Ciprofloxacin and Ceftazidime. Though we have not considered the anaerobes due to our limited facility, the literature showed the growth rate of anaerobes ranging from $20 \%-94 \%$. $^{11,14,15}$

Penicillin used to be the antibiotic of choice for the treatment of peritonsillar abscess, but in recent years the emergence of beta-lactamase-producing organisms and anaerobes has required a change in antibiotic choice. 16-18

\section{CONCLUSIONS}

Streptococcus pyogenes and Staphylococcus aureus are the most commonly associated organisms in peritonsillar abscess (PTA). Penicillin is commonly used antibiotic for PTA but emergence of beta lactamase producing organisms and increasing association of anaerobes should be considered in management of peritonsillar abscess. 


\section{REFERENCES}

1. Petruzzelli GJ, Johnson JT. Peritonsillar abscess. Why aggressive management is appropriate. Postgrad Med. 1990 Aug;88(2):99108.

2. Spires JR, Owens JJ, Woodson GE, Miller RH. Treatment of peritonsillar abscess. A prospective study of aspiration vs incision and drainage. Arch Otolaryngol Head Neck Surg. 1987 Sep;113(9):984-6.

3. Herzon FS. Peritonsillar abscess: incidence, current management practices, and a proposal for treatment guidelines. Laryngoscope. 1995Aug;105(suppl74):1-17.

4. Raut VV, Yung MW. Peritonsillar abscess: the rationale for interval tonsillectomy. Ear Nose Throat J. 2000 Mar;79(3):2069.

5. Maharaj D, Rajah V, Hemsley S. Management of peritonsillar abscess. J Laryngol Otol. 1991 Sep;105(9):743-5.

6. Forbes BA, Sahim DF, Weissfeid AS. Bailey and Scott Diagnostic microbiology. 11th ed. NewYork: Mosby; 1998. p.148-68.

7. Forbes BA, Sahim DF, Weissfeid AS. Bailey and Scott Diagnostic microbiology. 11th ed. NewYork: Mosby; 1998. P. 298-315.

8. Performance standards for antimicrobial disc susceptibility tests. Approved standard M2-A9. 9th ed. Wayne, Pennsylvania: Clinical and Laboratory Standards Institute; 2006.

9. Ong YK, Goh YH, Lee YL. Peritonsillar infections: local experience. Singapore Med J. 2004 Mar;45(3):105-9.

10. Macnamara M. Acute and chronic pharyngeal infection. In: Gleeson M, editor. Scott-Brown's Otorhinolaryngology, Head and Neck Surgery. 7th ed. London: Hodder Arnold; 2008. p. 1981-2024.
11. Jokipii AM, Jokipii L, Sipila P, Jokinen K. Semiquantitative culture results and pathogenic significance of obligate anaerobes in peritonsillar abscesses. J Clin Microbiol. 1988 May;26(5):957-61.

12. Hall SF. Peritonsillar abscess: the treatment options. J Otolaryngol. 1990 Jun;19(3):226-9.

13 Snowd G, Campbell JB, Morgan DW. The microbiology of peritonsillar sepsis. J Laryngol Otol. 1991 Jul;105(7):553-5.

14. Brook I. Microbiology and management of peritonsillar, retropharyngeal, and parapharyngeal abscesses. J Oral Maxillofac Surg. 2004 Dec;62(12):1545-50.

15. Mitchelmore IJ, Prior AJ, Montgomery PQ, Tabaqchali S. Microbiological features and pathogenesis of peritonsillar abscesses. Eur J Clin Microbiol Infect Dis. 1995 Oct;14(10):8707 .

16. Brook I, Frazier EH, Thompson DH. Aerobic and anaerobic microbiology of peritonsillar abscess. Laryngoscope. 1991 Mar;101(3):289-92.

17. Parker GS, Tami TA. The management of peritonsillar abscess in the 90s: an update. Am J Otolaryngol. 1992 Sep-Oct;13(5):2848 .

18. Megalamani SB, Suria G, Manickam U, Balasubramanian D, Jothimahalingam S. Changing trends in bacteriology of peritonsillar abscess. J Laryngol Otol. 2008 Sep;122(9):928-30. 